Canadian Oncology

Nursing Journal

Revue canadienne

de soins infirmiers

en oncologie

Volume 29, Issue 3 • Summer 2019

elSSN: 2368-8076 


\title{
Supportive care experiences of rural women living with breast cancer: An interpretive descriptive qualitative study
}

\author{
by Joanne Loughery and Roberta L. Woodgate
}

\begin{abstract}
Breast cancer is the leading cause of female cancer in Canada. However, there is limited research on the health and supportive needs of rural women living with this illness. The purpose of this qualitative study was to arrive at an increased understanding of the supportive care experiences of rural women requiring treatment for invasive breast cancer who reside in rural Manitoba. To explore this phenomenon, an interpretive descriptive qualitative study was completed. Twenty women from four regional health authorities participated in the study. Data were collected using face-toface, semi-structured interviews and analyzed by content analysis through an interpretative qualitative lens. The findings presented emphasize a holistic overview of the experiences of women living with breast cancer that captured both the positive aspects of living rurally and the described rural challenges. Pragmatic recommendations have been generated from the study findings that have implications for nursing practice and service delivery.
\end{abstract}

Key words: breast cancer, supportive care, experiences, rural, qualitative, interpretive description

C ancer is a devastating illness regardless of where a person lives. Breast cancer is the leading cause of female cancer in Canada with approximately 26,300 Canadians diagnosed each year (Canadian Cancer Society [CCS], 2017). One in every five Canadians lives in a rural setting, accounting for $18.9 \%$ of the population (Statistics Canada, 2015). Canadian research on the rural breast cancer experience is limited, with only two previous studies noted in the literature (Gray, James, Manthorne, Gould, \& Fitch, 2004; Fitch et al., 2003). With the rising incidence in breast cancer in our country, it remains an important healthcare topic. Moreover, access to oncology expertise and services is limited in rural settings, therefore extensive travel and relocation is inevitable. By utilizing qualitative

\section{ABOUT THE AUTHORS}
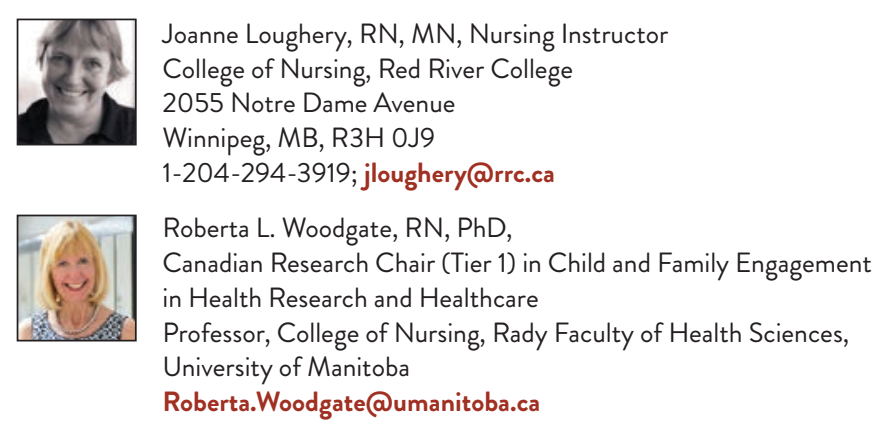

Roberta L. Woodgate, RN, PhD,

Canadian Research Chair (Tier 1) in Child and Family Engagement in Health Research and Healthcare

Professor, College of Nursing, Rady Faculty of Health Sciences, University of Manitoba

Roberta.Woodgate@umanitoba.ca

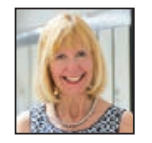

DOI:10.5737/23688076293170176 inquiry in this study, the voice of rural Manitoba women living with breast cancer has been captured and provides a theoretical foundation for intervention and opportunity for positive change that warrants consideration.

\section{BACKGROUND}

In Manitoba, approximately 860 women are diagnosed with breast cancer each year (CCS, 2017). Invasive, ductal carcinoma accounts for $80 \%$ of all breast cancer cases. As a result, multi-modality therapy that involves a combination of surgery, chemotherapy/biologic therapy, radiation, and long-term hormone treatment is required (Crane-Okada \& Loney, 2007; National Comprehensive Cancer Network [NCCN], 2014). The complexity of the experience increases for rural women due to lack of access to oncology services and fragmentation of healthcare available in rural settings. Cancer services in Manitoba include the following: specialized cancer service in the province capital; Western Cancer Centre in second largest city in Manitoba including multidisciplinary oncology services, chemotherapy, radiation and limited surgical procedures; 15 rural community cancer programs (CCP) that deliver chemotherapy, symptom management and follow-up care; and navigation, psychosocial oncology, and primary care in oncology programs in each regional health authority (CancerCare Manitoba [CCMB], 2000; 2017). Despite this, the need to travel to the urban centre is inevitable because surgery, breast reconstruction and radiotherapy are not available in most rural settings.

The supportive care framework is a tool utilized by health professionals to conceptualize the needs of individuals living with cancer (Fitch, 2009). Cancer is an unanticipated life event. It can create feelings of anxiety and vulnerability when basic human needs are not met. Individuals have a wide range of needs including the physical, social, psychological, emotional, spiritual, informational and practical domains. As treatment complexity increases, so will needs in each of these domains (Fitch, 2009). The supportive care framework was used to guide the research process in this study.

A review of the literature was completed and provided a foundation to explore the phenomenon of supportive care and the experiences of rural women living with breast cancer. Initially, an overview of breast cancer care across the illness trajectory was completed. It was apparent from the literature that each phase of illness has unique challenges and treatment-related problems (Crane-Okada \& Loney, 2007; Fitch, 2009; NCCN, 2014).

Next, the needs of all women living with breast cancer were explored. Needs exist in each domain of the supportive 
care framework and no two individuals have exactly the same response to cancer and its treatment. However, common patterns within the breast cancer and rural experience are apparent. Some challenges related to rural living that are featured in the literature include: physical symptoms exacerbated by travel; limited access to family doctors; isolation from oncology expertise; social disruptions related to relocation; lack of privacy in rural communities; additional out-of-pocket costs related to travel and relocation; and treatment decisions made based on inability to travel (Fitch et al., 2003; Gray et al., 2004; Loughery \& Woodgate, 2015; Matthews, West \& Buehler, 2009; Pesut, Robinson, Bottorff, Fyles, \& Broughton, 2010). However, there are also many positive aspects to rural living noted in the literature including: extension of support and close community ties; hardiness and resilient characteristics that foster effective coping; physical beauty and peaceful living; and close access to religious affiliations (Compas \& Luecken, 2002; Pesut et al., 2010; Rogers-Clark, 2002; Winter \& Lee, 2010).

Lastly, other models of oncology care from a national and international perspective were explored. It became clear that decentralization of cancer services into rural setting would improve services for all people living with cancer, but there is still room for improvement (Adams et al., 2009; Carlow, 2000; Clinical Oncology Society of Australia, 2006; National Institute for Clinical Excellence, 2004). With this in mind, this research project was initiated.

\section{PURPOSE}

The primary purpose of this qualitative study was to arrive at an increased understanding of the supportive care experiences of adult women requiring treatment for invasive breast cancer who reside in rural Manitoba. Findings were described from detection to the end of the treatment phase of illness and recommendations were generated directly from the study findings.

\section{METHODS}

Interpretive description, a qualitative investigation of clinical phenomenon, was utilized for this study (Thorne, 2008). This methodology provided a basis to understand the illness experience and how nurses make a difference and, ultimately, impact the lives of people living with illness. Interpretive description is rooted in a naturalist orientation, which honours individual experiences and, at the same time, allows for shared realities. These shared realities were used to generate an interpretive description capable of informing practice (Thorne, 2008). Ethics approvals from the Nursing Research Ethics Board, four health authorities and CCMB were obtained.

Inclusive criteria included the following: 1) adult women who were able to speak, read and write in English with no cognitive impairment; 2) a diagnosis of invasive breast cancer following completion of multi-modality treatment within the last three years; and 3) women who reside in rural or northern areas of the Manitoba. Women were excluded from the study if they had stage IV, relapsed or progressive illness, which results in different treatment plans. Purposive sampling was utilized to capture the essence of the rural breast cancer experience through the shared characteristics (Streubert \& Carpenter, 2011). A naturalistic setting of the participant's choice was honoured, which required more than $10,000 \mathrm{~km}$ of travel across the province.

Data were collected through face-to-face interviews, demographic questionnaires, field notes and journaling. Twenty first interviews and 12 second interviews were completed. At this point, the authors determined that repetition of data was occurring and no new information was surfacing (Streubert \& Carpenter, 2011). Therefore, the remaining eight second interviews were not completed.

The women participated in open-ended, semi-structured interviews, which were recorded and transcribed verbatim. All the interviews were coded and analyzed through directed content analysis. This approach was used to validate the theoretical framework by looking at relationships within the coded data (Zhang \& Wildemuth, 2009). Both authors iteratively reviewed all the raw data until interpretive descriptions were developed and then deductively shaped the data into categories within the domains of the framework. At the conclusion of the study, each of the study participants was provided with a summary of the findings with an open invitation for clarification. Methodological rigor was addressed through prolonged engagement with the participants, returning to the participants by way of member checking, peer review and debriefing, and documenting a detailed audit trail (Streubert $\&$ Carpenter, 2011).

\section{DEMOGRAPHIC AND QUALITATIVE FINDINGS}

\section{Demographic Findings}

Demographic data include participant descriptions, treatment modality data, and regional and travel demographics. Demographic data were collected through questionnaires and analysis of each interview.

Participant Description. Five women from each health authority, representing four regions of the province, participated in the study. The age ranged from 34-85 years with the majority of women between the ages of 46-65 years. Eleven women were married while the remainder were single, widowed or separated. Six women lived alone and the remainder lived with their spouse, adult children, children or grandchildren. Ten women were employed at the time of their diagnosis and five women worked through various stages of their treatment. The women who were employed worked in a variety of settings including healthcare, retail, office or home-based business. Half the women had completed postsecondary education and the remainder obtained Grade 12 or less. The majority of the women were Caucasian.

Treatment Modality Demographics. Ten women received a lumpectomy and the remainder received a unilateral or bilateral mastectomy with or without reconstruction or implant. All women required 4-8 cycles of chemotherapy and seven women required an additional year of biologic therapy. All of the women completed involved-field radiotherapy and 16 had initiated hormone therapy. 
Regional and Travel Demographics. Eleven women lived or were close to a city with a population of 7,500 people or greater, while the remainder lived or were close to a town with a population less than 7,500 people. Sixteen women had a rural CCP in their community and four women were required to travel to access chemotherapy services. The travel distance to Winnipeg by these women was between $62-1,100 \mathrm{~km}$ and to Brandon was from $15-182 \mathrm{~km}$. The women from one region had access to additional services in Brandon including surgery, chemotherapy and radiation. The primary mode of travel was land, but four women from the northern region utilized air travel and had access to financial compensation through Northern Patient Transport Services. Seventeen women had surgery in Winnipeg and the remainder in Brandon. Eighteen women had chemotherapy in a rural CCP and the remainder in Winnipeg. Sixteen women had radiation in Winnipeg and the remainder in Brandon.

\section{Supportive Care Qualitative Findings}

The findings have been categorized according to the domains in the supportive care framework (Fitch, 2009). A summary of the most common experiences is featured, however, individual variations are not captured.

Physical Domain. The physical domain was very demanding for the women. The side effects varied based on the mode of therapy. Chemotherapy was the most challenging treatment modality. Additionally, physical challenges occurred in relation to living rurally. During initial diagnosis, challenges occurred related to the travelling mammogram program or obstacles posed delays in diagnosis related to variables such as winter driving. During treatment, travel continued to be a challenge related to worsening physical symptoms such as fatigue, pain or skin reactions. Additionally, travel safety concerns were apparent related to symptoms such as overwhelming fatigue, "chemo fog" or not being able to wear a seatbelt following surgery. One single woman who drove herself daily to her radiation treatments, talked about travel safety as a primary concern.

But you know, with the chemo fog and being tired from radiation, you don't think properly, I should not have been driving.

Access to experienced and qualified health professionals in non-cancer settings or limited rural emergency services to manage complex physical needs was also a worry for these women. As a result, additional burdens on family and friends for travel or care for complex physical needs resulted. However, the positive roles of the rural cancer program and home care services were described and helped many transition through the physical demands that were encountered.

Informational Domain. Information was retrieved from a variety of health providers including family doctors, oncology specialists, hospital staff, urban and rural nurses, nurse navigators and other interdisciplinary professionals. A number of factors impacted the ability to process the information including escalating fears, long travel days, condensed appointment schedules, complex information, or lack of a support person available to attend the appointment. A 50-year-old woman, who lived a considerable distance from the urban centre, talked about enduring multiple appointments with specialists in a short period of time. She subsequently felt pressured to make a treatment decision she later regretted.

Everything just happened so fast, I started thinking like, oh, what happened if I feel like .... because I don't have breasts, like everything just happened so fast that I wouldn't have gotten reconstructed...I had to make up my mind right there when they were telling us and it's like uh, like it was all so fast.

Access to family doctors appeared to be fragmented across the province and communication between rural and urban sites also provided challenges. Additionally, it became apparent that rural providers or hospital staff with limited knowledge about cancer-related problems became an additional challenge. The role of the nurses and staff at the rural cancer centre was vitally important to meet the informational needs of the women, but there was a lack of physical space or privacy for personal discussions in the rural sites. The use of nurse navigators or access to other interdisciplinary health professionals was limited and would have proved to be very useful. Many informational resources or other supportive services were utilized and both positive and negative opinions on the usefulness were described.

Social Domain. A vast scope of social networks including immediate or extended family, friends or community members was described and utilized at different points in time during the experience. The scope of supportive networks also varied based on marital status or available family or friends in the communities. Living alone was viewed both positively and negatively, but did pose practical challenges. Additionally, health providers, especially nurses in the rural setting, were an important source of support for the women and viewed positively.

The love of rural life was apparent. A strong sense of community atmosphere and peaceful living was described by the women. However, it also posed specific obstacles such as lack of anonymity, invasion of privacy, or communication challenges with others and would often lead to feelings of isolation. One woman who lived in a small community her entire life felt her diagnosis was a very personal experience and, for this reason, isolated herself from others in the community for the duration of her treatment. At the time of the interview, a year following treatment, she was just starting to re-integrate into public life.

Yeah, 'cause everyone knows everyone's business. That's the way it is. If you are a private person and you want to keep things private, it's pretty tough... and usually it gets out and it goes like wildfire.

Furthermore, relocation for treatment, physical challenges of illness and avoidance of public places or other people were also found to be isolating. A number of women talked about being approached by either complete strangers or distant acquaintances asking them very personal questions 
about their illness or making comments that were upsetting or made them feel very uncomfortable. Other unique obstacles to rural life included personal connections with caregivers or having treatment with known community members. Some women talked about feeling very comfortable in the close-knit supportive environment of the CCP, whereas others were very uncomfortable in that intimate, personal setting.

Practical Domain. Many practical challenges existed and complicated the experience for these women. Managing basic daily activities when faced with other chronic illness became challenging. Other daily household chores or provision for dependent family members also required additional support. Some women had the needed support to help them manage, but others were forced to hire additional help or had no choice but to manage independently. This would often include the care of large acreages or farmyards. The burden of extensive travel, relocation, employment challenges and financial losses were significant findings in this study. Multiple trips to an urban centre, hazardous driving conditions, highway construction or navigating an unfamiliar city added to the complexity of the experience. Accommodating appointment schedules around travel was highly appreciated. However, limited diagnostic services in rural settings increased their demanding schedules.

Additionally, the northern women had unique challenges related to air and bus travel and were forced to relocate for radiation because of where they lived. Some women had the option to commute or relocate for radiation, but the decision significantly added to either the cost of relocation or the burden of travel. Supportive and affordable accommodation or staying in other comfortable lodgings proved to enhance the experience for the relocated women. However, some were not provided with this opportunity. The financial burden of cancer was apparent. Employment challenges, lost income and out-of-pocket costs were the primary issues contributing to this burden. One single woman talked about financial challenges prior to her diagnosis and how not being able to work and the additional out-of-pocket costs associated with rural life were financially devastating for her. This ultimately led to her moving out of her current living space to a more affordable option.

It cost me a lot because of the fact that I had to leave work.

I didn't have my income coming in anymore, so I, I went through pretty much everything I had in my savings.

Out-of-pocket costs would include fuel, accommodation, taxi rides, meals, household care, parking or purchasing additional medical supplies. Although financial support from friends, family and communities was appreciated, the out-ofpocket cost associated with living rurally remained noteworthy.

Emotional Domain. Many emotions were described during the course of the interviews. Shock, disbelief, denial, fear, uncertainty, anger, guilt and feelings of anxiety were discussed. These are very common feelings when presented with a diagnosis of cancer. However, many of these emotions were heightened by the rural experience. Increased emotions around relocation, challenges with inexperienced or insensitive care providers, and additional costs were featured. Travel burden also added to the ongoing emotional stress the women were experiencing. One woman described an incident where she drove hours to receive her biopsy results, filled with anxiety. Following a lengthy wait to see the specialist to receive her results, she then had to drive back to her home town alone filled with emotions. Many similar stories were illustrated during the interviews.

They are going to tell me that I have cancer and I have to drive here and then wait for two hours. I was kind of angry about that.

There were many descriptions about ways to release and cope with the complex emotions the women endured. Crying, venting or spending time alone were often useful. Additionally, using distraction such as music, yoga, meditation or gardening was also described. Many found solace in family, friends, co-workers, congregation members, care providers and other cancer survivors to help them through the experience. Moreover, the impact of close-knit communities and highly personalized care in the rural cancer settings also helped facilitate their emotional needs. Family response to illness was not thoroughly explored in this study, but it was apparent that a cancer experience is a family affair and separation from family related to relocation or travel was emotionally difficult.

Psychological Domain. Unmet needs in the psychological domain were described such as loss of control over the illness experience, altered body image related to hair loss or loss of a breast, or decreased self-esteem. Finding ways to cope with all these additional unmet needs was necessary. Gaining personal control by changing perspective on the illness or looking for positive opportunities as a result of the illness proved to be helpful for some. Additionally, utilizing distractions or taking a step-by-step approach to the illness also helped lessen these overwhelming feelings. One woman described many emotions related to her cancer journey and the only way she felt in control was by focusing on the here and now. In this situation, she chose to travel for more than six hours per day over the course of her radiotherapy treatments with the support of her spouse.

I guess you just cope the best you can... Like you almost kind of get into this mode where, well I have to do this, this is what I'm focusing on right now, this is what I have to do, like going back and forth to the city every day, that was what you had to do.

Focusing on staying well and fit proved to be very important and would result in some women feeling more energized and in control. Several attributes were also apparent such as resilience, self-reliance and optimism, which also fostered effective coping and promoted a positive self-esteem.

Spiritual Domain. As difficult as the journey was, it was often described as a time for discovery. For some of the women, facing their own mortality remained a very fearful process, 
but many found different ways to cope with this ongoing uncertainty. Some found solace and comfort in their spiritual practices or prayer, yet were challenged to attend services because of other physical or social demands. One woman described her sole strength was her faith in God. However, she did isolate herself from her congregation because she felt shame from her diagnosis and found it very difficult to talk to others.

I couldn't have done this without God and prayer. And that's where I get all my strength from, because on my own I was weak, and I had to really count on him, especially going into the chemo.

Some women described the journey as just another hurdle in their lives. Others were unable to explain why the diagnosis happened to them and, therefore, moved through a process of finding a deeper meaning to the illness. Meaning and gaining new perspectives, such as developing a new and deeper sense of self or family, feeling thankful for life and each new day, or developing new and existing connections with others were described. Some women talked about this as a life-changing journey.

\section{DISCUSSION}

Our study provided an in-depth picture of the supportive care experiences of women requiring treatment for invasive breast cancer who reside in rural Manitoba. Guided by Fitch's (2009) holistic supportive care framework, we detailed both the positive and negative aspects of living rurally when faced with illness. We anticipate these findings should be of interest to nurses who work with rural people living with cancer. The findings are strongly supported by the literature and, therefore, provide grounding to develop tailored interventions to improve cancer services for rural individuals living with cancer.

Nurses working in oncology are positioned to provide professional leadership at many levels of the health system and pave the way to improve services for rural people living with cancer (Canadian Association of Nurses in Oncology [CANO], 2001). The role of nursing in both urban and rural sites is highly valued and it is imperative that nurses are familiar with the rural implications when faced with an individual from a rural setting who is diagnosed with cancer.

Additionally, the women who used navigation services described that role as essential. However, navigational service was underutilized. Nurse navigators are positioned to provide patient and family-centred services, provide ongoing patient education, act as advocates, and serve as a liaison between oncology specialists, primary care physicians, rural cancer practitioners and treatment staff. They also facilitate the web of diagnostic procedures and multiple treatments while providing overall case management through each phase of the cancer journey (Farber, Deschamps, \& Cameron, 2002). This coordination of care throughout the treatment trajectory is essential for people living with cancer and becomes more crucial for our rural cancer population because of fragmentation of services. Concepts such as ambulatory oncology, early discharge from acute settings, complex treatment plans and early follow-up in primary care settings can make the cancer experience that much more confusing for patients and their families. Nurses practising in both cancer and non-cancer settings can provide a link to continuous and coordinated care while considering the complex rural issues that were presented in the findings of this research study.

Recommendations (Appendix A, Table 1) generated from this study could enhance service delivery in rural areas. These recommendations could provide the opportunity to prevent delay in diagnosis, improve wait times, decrease travel demands, promote family-centred care, enhance support and services, and facilitate positive adaptation to the cancer experience. Many challenges exist in rural healthcare, which are highlighted in a report mandated by the Government of Manitoba in 2017 (Peachey, Tait, Adams, \& Croson, 2017). Recommendations from this report that could potentially improve services for people living in rural areas include increased interdisciplinary care in primary care settings, enhanced rural home care supports, increased use of telehealth services, and increased funding for travel for people living in geographically isolated areas. Alternatively, re-alignment and centralization of emergencies and paramedic services across the province could be problematic (Peachey, 2017).

Additionally, CCMB 2016-2021 Strategic objective \#5 (CCMB, n.d.) highlights the need to improve care for underserved populations. Provision of new and enhanced access to services for residents of geographically-isolated areas was identified as a priority. The authors of this manuscript challenge nurses in Manitoba and across the country to become familiar with any recent policy documents from the government and to utilize this as leverage to initiate change. The recommendations presented in this study are not new ideas, but are still valid and should be considered as a priority.

There are study limitations that warrant discussion. First, the age differentiation of the women was not thoroughly explored. It did not become apparent during the interview process that there was a major difference in the experiences based on age. However, age and lived breast cancer experience certainly warrant further inquiry. Moreover, people who live in remote areas were not captured in the study and it could be assumed that the challenges they face would be increasingly complex. Additionally, populations such as men living with illness, different cultural ethnic backgrounds, long-term survivors, advanced or palliative diagnosis and family caregivers were not featured. These indications provide a path for future research to gain insight into the depth of the rural experience in other populations.

However, the findings generated from this research provide rich descriptions and insight into the rural experience and illuminate areas where change can and should happen. It will take a collaborative and collective effort to drive the vision to improve supports and service through the words of the women. Nurses in collaboration with other health professionals who work with rural cancer patients are positioned to provide leadership in this regard. 


\section{CONCLUSION}

The study findings feature the supportive care needs of rural women living with breast cancer from diagnosis to the end of the treatment journey. The findings and recommendations should be considered from a national perspective, as similar models of cancer service and geographic isolation in cancer populations remain as challenges across the country. We cannot change the fact that people live in rural areas of the country and specialized oncology services are primarily located in large urban settings. However, nurses and other care providers need to consider the unique features and implications that people face with a diagnosis of cancer who live rurally. There is still more that can be done to improve supports and services and enhance the quality of life and care for people living with this difficult illness in rural areas. The findings from this study provide insight into an important healthcare topic and a starting point to create awareness and change.

\section{APPENDIX A}

Table 1. Recommendations for Service Delivery

- Increase diagnostic procedures in rural settings.

- Encourage specialist site visits in rural communities.

- Record sessions with specialist.

- Increase awareness and utilization of navigation programs.

- Enhance rural primary care services and linkage to CCMB.

- Increase use of interdisciplinary healthcare team in rural areas.

- Increase use of telehealth services.

- Improve emergency care in rural settings.

- Encourage family escort during travel to enhance family-care giving.

- Consider discharge times and appointment schedules around travel.

- Promote socially directed supportive services such as meal service, driver programs or education or peer support programs in rural settings.

- Expand supportive relocation facilities and accommodations in urban settings.

- Improve aesthetics and private spaces in rural cancer settings.

- Expand oncology educational opportunities for practising generalist.

\section{REFERENCES}

Adams, P., Hardwick, J., Embree, V., Sinclair, S., Conn, B. \& Bishop, J. (2009). Models of cancer services for rural and remote communities. Retrieved from http://www.cancerinstitute.org.au/media/70218/ web09-83-02_literature_review_models_cancer_services_rural_ and_remote_communities.pdf

Canadian Association of Nurses in Oncology. (2001). Standards of care, roles in oncology nursing, and role competencies. Retrieved from http://www.cano-acio.ca/ ASSETS/DOCUMENT/Practice/ standards\%20of\%20care.pdf

Canadian Cancer Society. (2017). Canadian cancer statistics 2017: Special topic: Pancreatic cancer. Retrieved from http://www.cancer. ca/ /media/cancer.ca/CW/cancer\%20information/cancer\%20 101/Canadian\%20cancer\%20statistics/Canadian-Cancer-Statistics2017-EN.pdf?la=en

CancerCare Manitoba. (2000). CCP program directory. Retrieved from http://www.cancercare.mb.ca/home/patients_and_family/ treatment_services/treating_patients_in_rural_manitoba/ rural_program_directory/

CancerCare Manitoba. (2017). Current programs. Retrieved from http://www.cancercare.mb.ca/home/patients_and_family/patient_ and_family_support_services/current_programs/index.cfm

CancerCare Manitoba. (n.d.) Delivering excellence: 2016-2021 Manitoba cancer plan. Retrieved from https://www.cancercare.mb.ca/export/ sites/default/About-Us/.galleries/files/corporate-publications/ Manitoba_Cancer_Plan_2016-2021.pdf

Carlow, D. R. (2000). The British Columbia cancer agency: A comprehensive and integrated system of cancer control. Health Quarterly, 3(3), 31-45. doi:10.12927/hcq.16755.

Clinical Oncological Society of Australia. (2006). Mapping rural and regional oncology services in Australia. Retrieved from https://www. cosa.org.au/media/1067/cosa_paper_mapping-regional-oncologyservices_2006.pdf

Compas, B.E., \& Luecken, L. (2002). Psychological adjustment to breast cancer. American Psychological Society, 11(3), 111-114.

Crane-Okada, R., \& Loney, M. (2007). Breast Cancer. In M.E. Langhorne, J.S. Fulton, \& S.E. Otto (Eds.), Oncology nursing ( $5^{\text {th }}$ ed., pp. 101-124). St. Louis: Mosby Elsevier.

Farber, J.M., Deschamps, M., \& Cameron, R. (2002). Investigation and assessment of the navigator role in meeting the informational, decisional and educational needs of women with breast cancer in Canada. Retrieved from http://publications.gc.ca/collections/ Collection/H39-663-2002E.pdf

Fitch, M. I., Gray, R. E., McGowan, T., Bunskill, I., Steggles, S., Sellick, S., Bezjak, A., \& McLeese, D. (2003). Traveling for radiation cancer treatment: Patient perspectives. Psycho-Oncology 12, 664-674. doi: 10.1002/pon.682

Fitch, M. I. (2009). Supportive care framework: Theoretical underpinnings (1st ed.). In M.I. Fitch, H.B. Porter, \& B.D. Page (Eds.), Supportive care framework: A foundation for person-centred care (pp. 11-29). Pembroke, ON: Pappin Communications.

Gray, R.E., James, P., Manthorne, J., Gould, J., \& Fitch, M.I. (2004). A consultation with Canadian rural women with breast cancer. Health Expectations, 7, 44-50.

Loughery, J., \& Woodgate, R.L. (2015). The supportive care needs of rural individuals living with cancer: A literature review. Canadian Oncology Nursing Journal, 25(2), 157-166. doi:10:5737/23688076252157166

Mathews, M., West, R., \& Buehler, S. (2009). How important are outof-pocket costs to rural patients' cancer care decisions? Canadian Journal of Rural Medicine, 14(2), 54-60.

National Comprehensive Cancer Network. (2014). Breast cancer. Retrieved from http://www.nccn.org/professionals/physician_gls/ pdf/breast.pdf 
National Institute for Clinical Excellence. (2004). Guidance on cancer services: Improving supportive and palliative care for adults with cancer. Retrieved from http://www.nice.org.uk/guidance/csgsp/ evidence/supportive-and-palliative-care-the-manual-2

Peachey, D., Tait, N., Adams, O., \& Croson, W. (2017). Provincial clinical and preventative services planning for Manitoba: Doing things differently and better. Retrieved from https://www.gov.mb.ca/health/ documents/pcpsp.pdf

Pesut, B., Robinson, C.A., Bottorff, J.L., Fyles, G., \& Broughton, S. (2010). On the road again: Patients' perspectives on commuting for palliative care. Palliative and Supportive Care, 8, 187-195. doi:10.1017/S1478951509990940.x.

Rogers-Clark, C. (2002). Living with breast cancer: The influence of rurality on women's suffering and resilience. A postmodern feminist inquiry. Australian Journal of Advanced Nursing, 20(2), 34-39.
Statistics Canada. (2015). Canada's rural population since 1851. Retrieved from http://www12.statcan.gc.ca/censusrecensement/2011/as-sa/98-310-x/98-310-x2011003_2-eng.cfm

Streubert, H. \& Carpenter, R.D. (2011). Qualitative research in nursing: Advancing the humanistic imperative. 5th Ed. Philadelphia: Lippincott Williams \& Wilkins.

Thorne, S. (2008). Interpretive description. Walnut Creek, CA: Left Coast Press.

Winter, C.A., \& Lee, H.J. (2010) Rural nursing: Concepts, theory and practice (3rd ed.) Springers Publishing Company, LLC: New York.

Zhang, Y. \& Wildemuth, B.M. (2009). Qualitative analysis of content. In B. Wildemuth (Ed.) Applications of social research methods to questions in information and library science, p. 308-391. Westport, CT; Library Limited. 